\title{
Mineração
}

\section{Concentração de itabirito silicoso da Mina do Sapecado/Complexo Pico - MBR, empregando o classificador hidráulico de fluxo transversal "crossflow"}

\author{
Hely Simões Gurgel \\ Eng./MBR, Mestrando do PPGEM/DEMIN/EM/UFOP \\ E-mail:hsg@caemi.com.br \\ Rosa Malena Fernandes Lima \\ Dre., Professora Adjunta, PPGEM/DEMIN/EM/UFOP \\ E-mail:rosa@demin.ufop.br \\ Maria Lúcia Magalhães de Oliveira \\ Dra.MCE Consultoria e Engenharia Ltda \\ E-mail:luciamo@uai.com.br
}

\section{Resumo}

Nesse trabalho, foi efetuada a concentração da fração granulométrica compreendida entre 0,15 e $1 \mathrm{~mm}$ do minério itabirito silicoso da mina do Sapecado do Complexo Pico/MBR, usando o classificador hidráulico de fluxo transversal "crossflow" em duas rotas distintas, efetuando a etapa de concentração "rougher" e a etapa de limpeza dos concentrados de espirais de Humphreys. Para uma alimentação constituída por 60,19\% de Fe e 11,81\% de sílica, foram obtidos concentrados, de ambas as rotas testadas, com teores de impurezas dentro ou muito próximos aos teores de mercado para os produtos comercializados pela empresa.

Palavras-chave: Minério de ferro, concentração gravítica.

\begin{abstract}
The hydraulic classifier "crossflow" was used to concentrate one iron ore sample between 0,15 to $1 \mathrm{~mm}$ size from the Sapecado MBR mine. The feed iron and silic contents were 60,19 and $11,81 \%$, respectively. This equipment was tested in two ways: in the rougher stage and in Humphreys concentrate cleaning stage. In both cases it was possible to produce concentrates inside or near the commercial MBR product specifications.
\end{abstract}

Keywords: iron ore, gravity concentration. 


\section{Introdução}

A escolha do método de concentração de minério de ferro (concentração gravítica, magnética e flotação) depende de alguns fatores, tais como teor da alimentação, mineralogia, malha de liberação, fatores econômicos, controle do teor do concentrado e efeito sobre a pelotização (Tovar e colaboradores, 1988 e Iwasaki, 1989).

Os separadores hidráulicos do tipo leito retardo têm sido usados em processamento mineral há muitos anos. Consistem de uma câmera de separação, onde a alimentação descreve uma trajetória descendente contra um fluxo de água distribuído ascendentemente. Tipicamente, esses equipamentos são usados para classificação, entretanto, se a distribuição granulométrica estiver dentro de limites aceitáveis, os separadores hidráulicos do tipo leito retardo podem ser usados para a concentração de partículas em função das diferenças de densidades entre as mesmas como é o caso do separador hidráulico de fluxo transversal "crossflow" (Dunn e colaboradores, 2000).

A diferença marcante entre o "crossflow" e os demais classificadores hidráulicos de leito retardado é que o mesmo utiliza um sistema de alimentação tangencial, de baixa velocidade para introduzir a polpa no equipamento. Essa configuração permite que a água alimentada atravesse o topo da unidade e vá para a câmara do "overflow” com menor distúrbio da água de fluidização dentro da câmera de separação. Para reduzir a velocidade do fluxo de alimentação, o sistema possui um anteparo antes da câmera de separação. O resultado desse sistema de alimentação é a eliminação de um excesso de água na câmera de separação. Kohmurench, 2000, e Heiskanen, 1993, fornecem detalhes sobre o princípio de funcionamento do equipamento.

Hearn (2003) relatou o emprego de separador hidráulico (Floatex) para concentração de minério indiano de ferro abaixo de $1 \mathrm{~mm}$, que produziu concentrado ("underflow") com teor de sílica abaixo de $1 \%$.
Os minérios hematíticos do complexo Pico da MBR produzem quatro produtos "lump ore", hematitinha , "sinter feed" e "pellet feed”. Em 2008, os mesmos estarão totalmente exauridos. Logo, será necessário processar os minérios itabiríticos para garantir a própria sobrevivência da empresa. Em estudos de beneficiamento dos itabiritos do complexo Pico/MBR, em escala de laboratório e piloto, verificou-se a necessidade de fragmentar os mesmos abaixo de $6,3 \mathrm{~mm}$. Essa operação, após o emprego de etapas de concentração, possibilita a produção de "sinter feed" e "pellet feed". Nesses estudos, ficou evidenciado que, para a faixa granulométrica do "sinter feed" grosso (1,0 a 6,3 mm), o método de concentração mais adequado é a concentração gravítica, utilizando jigagem. Para a faixa do "pellet feed fine" $(>0,15 \mathrm{~mm})$, a flotação é o método mais eficaz. Para a fração fina do "sinter feed”( $0,15 \mathrm{a} 1 \mathrm{~mm})$, torna-se fundamental o estudo de novas técnicas de concentração, para se atingirem as especificações de mercado, sendo este o propósito desse trabalho, que efetuou o emprego do separador hidráulico de fluxo transversal "crossflow" para concentrar a fração do "sinter feed" fino.

\section{Materiais e métodos}

Nesse trabalho, foi utilizada uma amostra de itabirito silicoso na faixa granulométrica compreendida entre 0,15 e 1 $\mathrm{mm}$, que era constituída de $84,5 \%$ de hematita e $6,8 \%$ de quartzo.

A análise granulométrica foi efetuada por peneiramento a úmido, usando a série Tyler de peneiras. Os teores de Fe foram determinados por via úmida (dicromatria), enquanto que os teores de silício, alumínio, fósforo e manganês foram determinados por espectrometria de plasma de acoplamento indutivo.

Os ensaios de concentração da amostra foram efetuados mediante o emprego do "crossflow" em uma etapa "rougher" e na etapa "cleaner", após a concentração da mesma em espiral de Humphreys.
Para a otimização dos ensaios de concentração, utilizou-se o planejamento fatorial de experimentos. As variáveis estudadas foram a vazão de água de elutriação (A - 3 e 18 l/min), altura da interface (B - 10 e $25 \mathrm{~cm})$, \% de sólidos na alimentação (C - 30 e 40\%) e taxa de alimentação (D - 500 e 1750 g/min.)

No segundo planejamento de experimentos, utilizando o "crossflow" para os dois fluxogramas testados, fixou-se a interface em $10 \mathrm{~cm}$ e fixou-se a \% de sólidos em 40. Foram variadas a vazão de elutriação (1,8 e $9 \mathrm{~L} / \mathrm{min})$ e a taxa de alimentação (250 e 750 g/min.).

As variáveis-respostas consideradas para esse trabalho foram a recuperação em massa $(X)$, teor de sílica no concentrado (Y) e índice de seletividade (Z).

\section{Resultados e discussão}

A distribuição granulométrica da amostra estudada, bem como dos produtos da separação, na espiral de Humphreys, e do "crossflow", na etapa “rougher” e "cleaner”, está apresentada na Tabela 1. Observa-se que cerca de $59 \%$ da alimentação encontrava-se na faixa granulométrica compreendida entre 0,15 e $1 \mathrm{~mm}$. Para o concentrado e rejeito da espiral de Humphreys, esses percentuais foram de 69 e $22 \%$, respectivamente. No caso do "crossflow" etapa "rougher", foram de 70,88\% e 13,46\%, respectivamente. Na etapa "cleaner" foi de $80,8 \%$ e $20,2 \%$, respectivamente no concentrado e no rejeito. Observam-se, também, maiores proporções de partículas menores que $0,037 \mathrm{~mm}(11,4 \%)$ e maiores que $1 \mathrm{~mm}(43,56 \%)$ no rejeito da espiral de Humphreys. Todas as partículas presentes no rejeito do "crossflow" etapa "rougher" encontravam-se abaixo de $1 \mathrm{~mm}$. Na etapa "cleaner" foram de $0,2 \%$. Cerca de $10 \%$ e 4,3\% das partículas presentes no rejeito do "crossflow" etapa "rougher" e da etapa "cleaner" encontravam-se abaixo de 0,038mm.

Na Tabela 2, está apresentada a análise granuloquímica do itaibirito silicoso, bem como os teores globais (ca- 
Hely Simões Gurgel et al.

beça calculada) de $\mathrm{Fe}, \mathrm{SiO}_{2}, \mathrm{Al}_{2} \mathrm{O}_{3}$ e $\mathrm{P}$, que foram de, respectivamente, 60,19; 11,81; 0,34 e $0,038 \%$. Observa-se que os teores de ferro, na fração compreendida entre 0,053 a 0,150 mm, são mais baixos, ocorrendo o inverso para o teor de sílica. Os teores de alumina são mais altos na fração acima de $0,3 \mathrm{~mm}$.

A fração responsável pelo maior percentual de sílica é a fração compreendida entre 0,15 e 0,3 mm. Como 78\% da amostra encontra-se acima de 0,15 mm, pode-se afirmar que a distribuição de ferro, alumina, fósforo e manganês ocorre, preferencialmente, nessa fração.

As equações que descrevem os efeitos das variáveis investigadas no primeiro planejamento de experimentos sobre as variáveis-respostas recuperação em massa (X), teor de sílica no concentrado (Y) e índice de seletividade (Z) são:

i) Etapa "rougher"

$X=50,6-29,4 A-3,31 D-2,53 B D+2,48$ ABCD...

$\mathrm{Y}=4,54-0,74 \mathrm{~A}-0,28 \mathrm{~B}+0,40 \mathrm{D}$

$Z=2,43-0,38 A-0,21 D$

ii) Etapa “cleaner"

$X=46,16-29,15 A-3,43 B-6,77 D+3,40 A D-2,18 B D+4,05 A B D-3,70 C D$.

$\mathrm{Y}=2,96-0,32 \mathrm{~A}-0,21 \mathrm{~B}+0,13 \mathrm{AB}+0,02 \mathrm{C}+0,02 \mathrm{ABC}+0,15 \mathrm{AD}+0,13 \mathrm{BD}-0,10 \mathrm{ABD}-$

$0,11 \mathrm{CD}+0,04 \mathrm{ACD}+0,07 \mathrm{BCD}$

$\mathrm{Z}=2,74-0,53 \mathrm{~A}-0,10 \mathrm{AB}-0,18 \mathrm{D}-0,12 \mathrm{BD}+0,18 \mathrm{ABD}$.

Tabela 1 - Distribuição granulométrica da alimentação, concentrado e rejeito da espiral de Humphreys e "crossflow".

\begin{tabular}{|c|c|c|c|c|c|c|c|}
\hline \multirow{4}{*}{$\begin{array}{c}\text { Tamanho } \\
(\mathrm{mm})\end{array}$} & \multicolumn{7}{|c|}{$\%$ Retida acumulada } \\
\hline & \multirow{3}{*}{ Alim. } & \multirow{2}{*}{\multicolumn{2}{|c|}{ Humphreys }} & \multicolumn{4}{|c|}{ "Crossflow" } \\
\hline & & & & \multicolumn{2}{|c|}{ Etapa "rougher" } & \multicolumn{2}{|c|}{ Etapa "cleaner' } \\
\hline & & Conc. & Rej. & Conc. & Rej. & Conc. & Rej. \\
\hline 1 & 19,11 & 13,53 & 43,56 & 23,63 & - & 16,52 & 0,19 \\
\hline 0,6 & 33,99 & 29,51 & 57,01 & 49,45 & 0,43 & 42,34 & 0,39 \\
\hline 0,3 & 49,64 & 48,86 & 60,70 & 72,46 & 0,54 & 72,54 & 0,51 \\
\hline 0,15 & 77,97 & 82,31 & 65,36 & 94,51 & 13,46 & 97,28 & 20,42 \\
\hline 0,106 & 85,14 & 89,80 & 69,31 & 98,22 & 48,20 & 98,46 & 59,34 \\
\hline 0,075 & 90,68 & 95,03 & 74,64 & 98,47 & 72,04 & 98,66 & 80,61 \\
\hline 0,053 & 93,78 & 97,50 & 79,94 & 98,62 & 83,19 & 98,73 & 90,70 \\
\hline 0,045 & 95,68 & 98,75 & 86,84 & 98,71 & 87,02 & 98,76 & 93,62 \\
\hline 0,038 & 96,67 & 99,17 & 88,58 & 98,82 & 90,24 & 98,80 & 95,66 \\
\hline$-0,038$ & 100 & 100 & 100 & 100 & 100 & 100 & 100 \\
\hline
\end{tabular}

Como pode ser visto na Figura 1 (a), obtém-se um concentrado com um teor de sílica em torno de 5,5\%, sendo este teor próximo ao produto denominado de "sinter feed 05" (os teores de Fe, $\mathrm{SiO}_{2}, \mathrm{Al}_{2} \mathrm{O}_{3}$ e $\mathrm{P}$ para o produto SF5 são 64,8, 5,0 , 0,85 e 0,042 ), com uma recuperação em massa satisfatória para essa etapa (74,1\%). O índice de seletividade foi igual a 2,64. Esse concentrado é passível de ser processado em uma etapa “cleaner”, objetivando abaixar o teor de sílica, assim como o rejeito pode ser aproveitado através de uma etapa "scavenger”, objetivando aumentar a recuperação global.

Para o segundo fluxograma, Figura 1 (b), através dessa rota, chegar-se-ia a um concentrado com teor de sílica dentro das especificações de mercado do produto denominado "sinter feed 3,5 (os teores médio de $\mathrm{Fe}, \mathrm{SiO}_{2}, \mathrm{Al}_{2} \mathrm{O}_{3}$ e $\mathrm{P}$ são respectivamente, $65,8,3,50,0,88$ e $0,046 \%$ ), recuperando $62,16 \%$ da massa da fração do "sinter feed fino".

\section{Conclusões}

Ficou comprovada a eficiência do classificador hidráulico de fluxo transversal "crossflow” para a concentração da fração granulométrica compreendida entre 0,15 e $1 \mathrm{~mm}$ do minério de itabirito estudado, pois foi verificado maior percentual de partículas nessa faixa em relação à alimentação para ambos fluxogramas testados.

Os teores de Fe, na amostra global, eram mais baixos na faixa granulométrica compreendida entre 0,053 e 0,15mm (55 a 60\%), ocorrendo o inverso para os teores de sílica (11 a 20\%).

A recuperação em massa da condição otimizada para o primeiro fluxograma testado, etapa "rougher" com o "crossflow", foi de $74,1 \%$. Os teores de ferro e demais impurezas estavam próximos das especificações de mercado de um dos produtos comercializados pela MBR (“sinter feed 05").

Para o segundo fluxograma testado com o "crossflow", efetuando a etapa de limpeza do concentrado da espiral 
Concentração de itabirito silicoso da Mina do Sapecado/Complexo Pico - MBR, empregando...

Tabela 2 - Análise granuloquímica do itabirito silicoso.

\begin{tabular}{|c|c|c|c|c|c|c|c|c|c|c|}
\hline \multirow{2}{*}{$\begin{array}{c}\text { Abertura } \\
\text { mm }\end{array}$} & \multirow{2}{*}{$\begin{array}{l}\text { \%Retida } \\
\text { Simples }\end{array}$} & \multicolumn{9}{|c|}{ Teores (\%) } \\
\hline & & $\mathrm{Fe}$ & $\mathrm{SiO}_{2}$ & $\mathrm{Al}_{2} \mathrm{O}_{3}$ & $\mathbf{P}$ & Mn & $\mathrm{CaO}$ & MgO & $\mathrm{TiO}_{2}$ & PPC \\
\hline $\begin{array}{l}\text { Cabeça } \\
\text { Calculada }\end{array}$ & & 60,19 & 11,81 & 0,34 & 0,038 & 0,020 & 0,054 & 0,032 & 0,031 & 0,85 \\
\hline 1,00 & 19,1 & 64,53 & 5,68 & 0,45 & 0,051 & 0,029 & 0,049 & 0,029 & 0,031 & 1,15 \\
\hline 0,60 & 14,9 & 64,92 & 5,29 & 0,42 & 0,062 & 0,028 & 0,050 & 0,033 & 0,031 & 1,09 \\
\hline 0,30 & 15,7 & 63,60 & 6,13 & 0,41 & 0,031 & 0,024 & 0,071 & 0,038 & 0,031 & 1,14 \\
\hline 0,15 & 28,3 & 55,06 & 19,71 & 0,24 & 0,020 & 0,014 & 0,048 & 0,032 & 0,031 & 0,50 \\
\hline 0,106 & 7,2 & 52,89 & 22,19 & 0,18 & 0,023 & 0,011 & 0,048 & 0,030 & 0,031 & 0,35 \\
\hline 0,075 & 5,5 & 56,28 & 17,08 & 0,19 & 0,021 & 0,010 & 0,052 & 0,033 & 0,031 & 0,62 \\
\hline 0,053 & 3,1 & 59,99 & 11,64 & 0,18 & 0,051 & 0,010 & 0,084 & 0,035 & 0,031 & 0,62 \\
\hline 0,045 & 1,9 & 60,54 & 10,47 & 0,20 & 0,052 & 0,011 & 0,056 & 0,034 & 0,031 & 0,72 \\
\hline 0,038 & 1,0 & 62,20 & 8,85 & 0,21 & 0,054 & 0,011 & 0,049 & 0,029 & 0,031 & 0,70 \\
\hline$-0,038$ & 3,3 & 63,34 & 6,43 & 0,72 & 0,072 & 0,026 & 0,064 & 0,034 & 0,032 & 1,48 \\
\hline
\end{tabular}

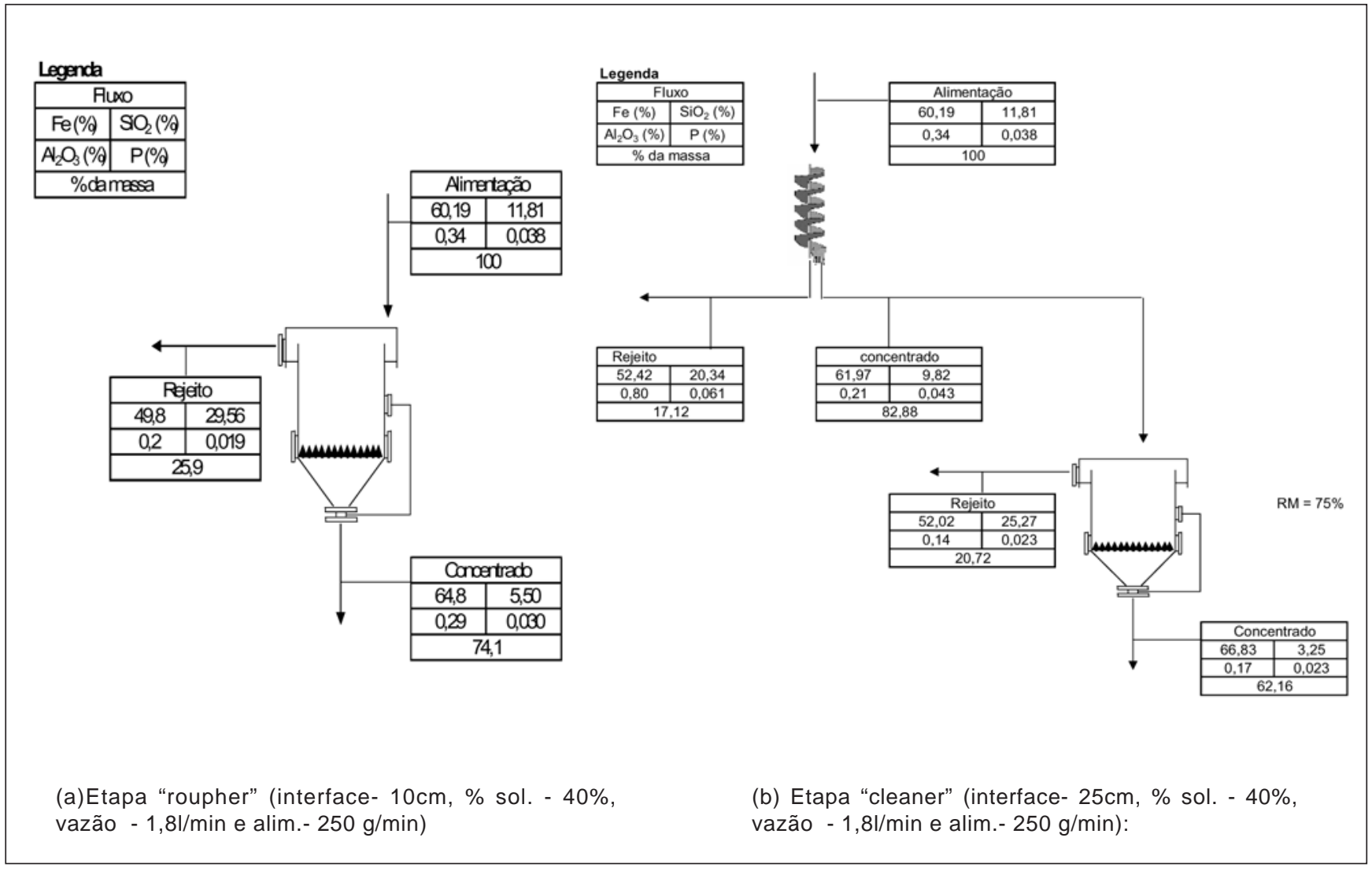

Figura 1 - Balanços de massa da concentração do minério itabirito silicoso, usando "crossflow" após otimização. 
Hely Simões Gurgel et al.

de Humphreys, gerou-se um concentrado final com teor de sílica igual a 3,25\%, recuperando-se $62,16 \%$ da massa.

\section{Referências bibliográficas}

DUNN P. L., STEWART S. O., KOHMUENCH J. N., CADENA C.A A hydraulic classifier evaluation: upgrading heavy mineral concentrates. In: SME Annual Meeting Salt Lake City, Utah - February 28 - March 1, 2000. p.3.

HEARN, S. The use of hindered settlers to improve iron ore gravity concentration circuits. Flórida, USA: 2003. p.936943.

HEISKANEN, K. Particle classification. London: Powder Technology Series, Chapman and Hall, 1993.
IWASAKI, I. Briding theory and practice in iron ore flotation. In: CNANDER, S., KLIMPEL, R. R. eds. Advances in coal and mineral processing using flotation. Inc, 1989, Chapter 20. p. 177-190.

KOHMURENCH N. J. Improving efficiencies in water-based separators using mathematical analysis tools. Faculty of the Virginia Polytechnic Institute, 2000. p.42-46. (Thesis).

TOVAR, P. E., NAVARRO, J., PASTRANA, J. M. Flotation of low-grade iron ore. In: FLORES, S. H. C., MOISAN, J. A. (Ed.) $2^{\text {nd }}$ Latin-American Congress on Froth Flotation, 1975, Conception, Chile. Froth Flotation. Amsterdan-Oxford-New York-Tokyo: 1988. p.215-231.

Artigo recebido em 02/03/2006 e aprovado em 14/07/2006.

\title{
REM - Revista Escola de Minas
}

\section{0 anOS divulgando CIÊNCIA.}

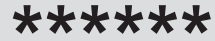

\section{REM: a mais antiga revista técnico- científica do setor mínero-metalúrgico.}

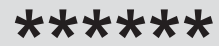 \\ www.rem.com.br

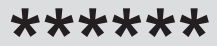

